\title{
Interactive comment on "Lateral transports of mass, inorganic nutrients and dissolved oxygen in the Cape Verde Frontal Zone in summer 2017" by Nadia Burgoa et al.
}

\section{Anonymous Referee \#3}

Received and published: 12 January 2021

Review of os-2020-98 "Lateral transports of mass, inorganic nutrients and dissolved oxygen in the Cape Verde Frontal Zone in summer 2017" Burgoa et al.

The manuscript describes the distribution of hydrographic, nutrients and oxygen concentrations along the sides of a large box that covers the upwelling area and CVFZ in the cape verde basin, along the Western Sahara and Mauritanian coasts during the summer of 2017 ( $\sim 26$ days).

Combining full depth CTD, XBT profiles and water samples the authors describe the distribution of water masses along the ships' track. The XBT data are used to increase the resolution of the temperature data in all transects and "optimal" interpolation pa- 
rameters are generated through the use of DIVA interpolation package. These are later used to interpolate the more sparse dataset of salinity (and hence density for mass transport estimates) and biochemical variables. Through the application of an inverse transport model the authors estimate mass and biochemical fluxes across the transects using observed (and climatological) geostrophic velocities as first guesses in an 8 layer distribution (defined approximately to separate the contributions by the major water masses in a similar manner to previous estimates for the region).

The main conclusions from this exhaustive exercise are that the distribution, characteristics and transports by each water mass are comparable to previous estimates already published for the region (and referenced in this manuscript). The detailed estimates per isoneutral layer are useful to compare with both climatological estimates and modelling products and I see value in the publication of these results.

\section{General comments}

The long time taken to complete the sampling introduces unavoidable aliases in the distribution of the variables and geostrophic velocities and this is reflected in the large errors associated with both biochemical and mass transports. The approach has been to use the annual mean estimate of the velocity variance at the reference level from GLORYS. Would it be better to use global model outputs instead that would have a higher resolution (more comparable to the resolution of the observations?). As for the validation, it would be very useful to compare the geostrophic transport estimates with the ship's ADCP data, at least for the SW and CW layers.

I would consider moving figures A8 and A13 earlier in the results section to provide a seasonal context to the observations. I would also welcome a brief description of the conditions in 2017 with respect to the other years to put the values into a wider context (maybe this can be done in the discussion instead).

I am curious to see the impact of using DIVA vs a standard krigging interpolation scheme. Would it be possible to include an example in the supplementary materials 
section? Do you think this would have an impact on the transport estimates?

Is there any reason why the water mass content was not quantified through the OpOSD timal Multiparameter Method? (OMP) (i.e. Zhou, P., Song, X., Yuan, Y., Cao, X., Wang, W., Chi, L., \& Yu, Z. (2018). Water mass analysis of the East China Sea and interannual variation of Kuroshio Subsurface Water intrusion through an Optimum Multiparameter method. Journal of Geophysical Research: Oceans, 123, 3723âĂŘ 3738.

Interactive https://doi.org/10.1029/2018JC013882)

Grammar/English There are some instances in which the text is difficult to follow due to complex descriptions and syntax that could benefit from the input of an english speaker. I know how difficult it is to summarise such a large dataset but adding every detail dilutes the main messages that one would like to convey. It is important that the descriptions be kept short with simple sentences to help the reader. This can be aid by anotating the figures (i.e. N, W, S and $\mathrm{E}$ in the title sections of the transect figures, depth labels in A1, special features/water masses in figs A5-6, major current names in $A 7$, features described in the text in A8 such as CVFZ etc.. )

The discussion and conclusions section would need to be revise in detail to make it easier to follow.

Detailed comments

P1L15 hinder (instead of hinders)

P2L1 Being a permanent upwelling area the CUF is always present (intensity and location might change). revise

P2L6 try and mimic SACW description (i.e. add location of where it forms)?

P2L15 IS modifiED

P2L14-21 Revise paragraph

P3L13-19 Include what sets the present manuscript apart from the papers cited. 
P4L22 - Add who provided the wind data (i.e. url or data provider)

P5L25.. I am afraid I couldn't understand this paragraph and what it meant. Could you OSD point in A1 what stations were in fact climatological nodes?

P7L5. This is an area where diapycnal mixing is significant, specially in the CW layers and in the confluence of NACW and SACW as here. Dismissing them from the inverse model might be another reason for the large errors in the estimates and the lack of mass conservation in the results. (Peña-Izquierdo, J., . On the circulation of the North Atlantic shadow zone 150., PeñaâĂŘlzquierdo, J., Sebille, E. van, Pelegrí, J.L., Sprintall, J., Mason, E., Llanillo, P.J., Machín, F., 2015. Water mass pathways to the North Atlantic oxygen minimum zone. Journal of Geophysical Research: Oceans 120, 3350-3372. https://doi.org/10.1002/2014JC010557)

P7L17 definitions for instead of "relationship between"

P7L29 included instead of "grouped"

P8L12-27 Revise text to make it clearer.

P8 I think this is where having the OMP results might help the description. This can complement the information in A11 and A12 too.

Clarify terminology when refering to eddy, meanders and filaments. Sometimes, their use in the text is confusing.

P9L18-19 revise sentence.

P24-25 Label of A11 and A12. Second NO3 should be PO4.

Interactive comment on Ocean Sci. Discuss., https://doi.org/10.5194/os-2020-98, 2020. 